\title{
El pensamiento computacional: ¿Una nueva forma de entrenar la memoria de trabajo?
}

\section{Computational thinking: A new way to train working memory?}

\author{
Eduardo Sanabria Zafra \\ Universidad de La Laguna, San Cristóbal de La Laguna, España \\ Noelia Rodríguez Rodríguez \\ Universidad de La Laguna, San Cristóbal de La Laguna, España \\ Andrés Eduardo Zerpa Pérez. \\ Universidad de La Laguna, San Cristóbal de La Laguna, España \\ Pedro Luis Prieto Marañón \\ Universidad de La Laguna, San Cristóbal de La Laguna, España \\ pprieto@ull.edu.es \\ María Ángeles Alonso Rodríguez \\ Universidad de La Laguna, San Cristóbal de La Laguna, España \\ maalonso@ull.edu.es
}

\begin{abstract}
Resumen
El pensamiento computacional es un concepto reciente que ha cobrado importancia en los últimos años. Se ha encontrado que entrenamientos basados en este concepto, son capaces de producir mejoras en diversas funciones cognitivas. En este trabajo se ha empleado un entrenamiento basado en el pensamiento computacional elaborado por el Aula Cultural de Pensamiento Computacional y la Fundación general de la Universidad de La Laguna. El entrenamiento se aplicó a un total de 49 alumnos de secundaria divididos en dos grupos: un grupo que se basaba en una metodología de aprendizaje guiada y otro basado en una metodología de aprendizaje por descubrimiento. El objetivo es comprobar si el entrenamiento es capaz de producir mejoras en las medidas de memoria de trabajo y si es más beneficioso para los participantes con un nivel de memoria de trabajo alto. La conclusión que se puede obtener de dicho estudio es que el entrenamiento, independientemente de la metodología que se aplique es capaz de producir mejoras en las diferentes medidas de memoria trabajo. Así mismo, no se encontró evidencia de que los participantes con un nivel de memoria de trabajo alto se beneficien más del entrenamiento en la mayoría de las pruebas, pero sí se encontró un beneficio mayor de estos en la prueba de figuras abstractas.
\end{abstract}

Palabras clave: memoria de trabajo, pensamiento computacional, entrenamiento.

Abstract

RED. Revista de Educación a Distancia. Núm. 63, Vol. 20. Artíc. 2, 30-04-2020

DOI: http://dx.doi.org/10.6018/red.401931 
Computational thinking is a new concept which has been adquiring relevance in the last few years. There are some evidences of trainings based on this new concept improving different cognitive abilities. In this paper, it has been used a training based on computational thinking, designed by "aula cultural de pensamiento computacional" and the general foundation of the University of La Laguna. This training was applied to 49 students of secondary obligatory education who had been divided into two groups: one group received the training with a methodology based in guide learning and the other group received the training based on selflearning methodology. The main objective of this paper is to prove that training can improve the measures of working memory and check if the participants who have a high level of working memory take more benefits of the training. The results show that the training based on non-specific methodology in computational thinking improved the measures of working memory. In addition, there was no evidence of a higher level of benefit of the training in most of the tasks in the participants who have a high working memory level.

Keywords: computational thinking, working memory, training.

\section{Introducción}

La sociedad actual está inmersa en las nuevas tecnologías, por lo tanto, es necesario que desde los colegios se trabaje el lenguaje digital a la vez que las materias tradicionales, puesto que éste incluye el uso de la programación, que ayuda al desarrollo de habilidades para la resolución de problemas como parte del pensamiento computacional (Llorens, 2015).

El pensamiento computacional es un concepto reciente. Su primera autora fue Wing (2006), el cual lo define como una herramienta fundamental que comprende la resolución de problemas, el diseño de sistemas y la comprensión de la conducta humana haciendo uso de los conceptos fundamentales de la ciencia de la informática, siendo útil para todo el mundo en su día a día, trabajen o no en el ámbito de la computación.

Según García y Mendes (2018, p.408 ) "El pensamiento computacional es una metodología activa de resolución de problemas en la que se utiliza una serie de elementos tales como la abstracción, la relación de patrones para procesar y analizar datos y para crear elementos reales o virtuales". En relación con esto, se han descrito una serie de componentes que forman parte del pensamiento computacional tales como: análisis ascendente; análisis descendente; heurística; pensamiento divergente; creatividad; resolución de problemas; pensamiento abstracto; recursividad; interacción; método de ensayo-error; métodos colaborativos, patrones; sinéctica y metacognición, aunque su presencia depende el tipo de tarea (Zapata, 2015).

Por estos motivos, en los últimos años se ha incrementado el interés en el concepto del pensamiento computacional, lo que ha motivado a que se intente implementar en el currículo académico en varios países. Sin embargo, la ausencia de un acuerdo a la hora 
de definir lo que es el pensamiento computacional dificulta encontrar una forma efectiva y consensuada de incorporarlo al mismo (Adell, Llopis, Esteve y Valdeolivas, 2019). Esto ha llevado a que frecuentemente en España, para desarrollar el pensamiento computacional, se trabaje utilizando procedimientos de programación mecánicos, favoreciendo por tanto, el estereotipo de que la programación es solo cosa de programadores (Zapata, 2015).

Se han llevado a cabo diferentes trabajos de cara a comprobar los beneficios que tienen los entrenamientos basados en el pensamiento computacional. En un trabajo llevado a cabo por Psycharis y Kallia (2017) se obtuvo que un entrenamiento basado en pensamiento computacional relacionado con las matemáticas producía mejoras en la percepción de eficacia en matemáticas, en la resolución de problemas y las habilidades de razonamiento. Por otro lado, en relación con lo anterior, se ha visto que la utilización del programa informático Scratch, contribuye al desarrollo de las habilidades lógicomatemática y a la mejoría del rendimiento académico (Korkmaz, 2016). Además, en un meta análisis sobre los efectos de transferencia del pensamiento computacional se ha encontrado que existe una transferencia positiva desde el pensamiento computacional a habilidades cognitivas, viéndose beneficiado especialmente el pensamiento creativo, el razonamiento y, en menor medida, el rendimiento académico (Scherer, Siddiq y Sánchez, 2018).

Por otro lado, se ha relacionado el pensamiento computacional con las habilidades mentales generales, así como con algunas aptitudes cognitivas específicas tales como las habilidades verbales, espaciales y de razonamiento inductivo (Román, Pérez y Jiménez, 2017). Así mismo, se ha visto que el entrenamiento en pensamiento computacional produce mejoras en las habilidades de metacognición (Scherer et al., 2018).

Como se planteó inicialmente, el pensamiento computacional es concebido como una metodología para la resolución de problemas que implica varias habilidades cognitivas. Uno de los componentes esenciales para realizar este tipo de tareas es la memoria de trabajo. Esta permite mantener activada la información durante un periodo de tiempo determinado, permitiendo así una integración adecuada de la misma para la resolución de problemas, así como para la construcción y la manipulación de los procesos mentales (Solaz y Sanjosé, 2008). Además es uno de los elementos básicos que interviene en cualquier proceso de aprendizaje (López, 2011); y un buen predictor en los inicios de la escolarización del éxito académico en etapas posteriores (Packiam y Alloway, 2010)

Con el fin de comprender mejor el funcionamiento de la memoria de trabajo, se han planteado varios modelos, pero uno de los modelos más aceptados a día de hoy es el modelo multicomponente de Baddeley y Hitch (1974). Inicialmente, en este modelo se plantea que la memoria de trabajo está formada por tres componentes: el bucle fonológico que permite la retención temporal de información verbal, la agenda viso-espacial la cual permite la retención temporal de la información de carácter visual y el ejecutivo central, que se encarga de controlar la atención durante la realización de una o varias tareas. Años después Baddeley añade un cuarto componente al modelo: el retén episódico, definido 
como: "un sistema de almacenamiento temporal de capacidad limitada que es capaz de integrar información de una variedad de fuentes" (Baddeley, 2000, p.421).

En los últimos años, se han desarrollado entrenamientos en memoria de trabajo que permiten producir mejoras en los déficits producidos en determinadas patologías. En relación con esto, Aguirre et al. en (2018) desarrollaron un tipo de entrenamiento de memoria de trabajo, empleando la tarea n-back, con el fin de mejorar la eficiencia cognitiva en pacientes con esclerosis múltiple remitente-recurrente. Los resultados obtenidos muestran que los participantes que recibían el entrenamiento de memoria de trabajo presentaban una mayor tasa de aciertos y un menor tiempo de respuesta que los participantes que no recibieron el entrenamiento. Además, se ha encontrado que un entrenamiento en memoria de trabajo, empleando la tarea n-back y aplicado a adolescentes, produce una disminución de los síntomas de ansiedad y de depresión, persistiendo estos resultados en el tiempo (Beloe y Darakshan, en prensa).

Por otro lado, la memoria de trabajo, no solo se puede mejorar con entrenamientos basados en tareas de este tipo, sino que se ha encontrado que un entrenamiento en mindfulness puede mejorarla o incluso producir efectos de transferencia a habilidades matemáticas (Gonzales, Fernández y Duarte, 2018).

También se han elaborado programas de entrenamiento computarizados p.e. RoboMemo, desarrollado por Cogmed Cognitive Medical Systems y utilizado por Klingberg et al. (2005) para el entrenamiento de la memoria de trabajo en niños con TDAH. Dicho programa se compone de una serie de tareas destinadas a trabajar dos componente de la memoria de trabajo: el componente verbal y el componente visoespacial. Los resultados obtenidos por Klingberg et al. (2005) mostraban una mejoría en la ejecución de dicha mejora en aquellos participantes que recibieron el entrenamiento, por lo que se concluía que la memoria de trabajo podía ser entrenada en niños con este trastorno.

En esta misma línea del uso de las nuevas tecnologías en la aplicación de entrenamientos, en este trabajo se ha empleado un programa de entrenamiento basado el pensamiento computacional, dirigido a alumnos de segundo de la ESO. Con la aplicación de este entrenamiento se pretenden comprobar las siguientes hipótesis: (1) El entrenamiento produce mejoras en las diferentes medidas de memoria de trabajo empleadas y (2) Los participantes con un alto nivel de memoria de trabajo se beneficiarán más del entrenamiento que los participantes con un bajo nivel de memoria de trabajo.

\section{Método}

\section{Entrenamiento}

El entrenamiento en pensamiento computacional ha sido elaborado por el Aula Cultural de Pensamiento Computacional y la Fundación general de la Universidad de La Laguna dentro del programa "Piens@ computacion@LLmente" financiado por el Cabildo de Tenerife con el programa Tenerife Educa 20-30. Este proyecto, el cual se 
encuentra en su tercera edición, está dirigido a fomentar y/o desarrollar el Pensamiento Computacional mediante una combinación de actividades y herramientas que permiten presentar y poner en práctica conceptos básicos en el ámbito del Pensamiento Computacional. Para dicho entrenamiento se cuenta con dos modalidades, $A$ y $B$.

La Modalidad A, se basa en un enfoque que permite desarrollar el pensamiento computacional desde la introducción de sus conceptos y principios básicos. Partiendo generalmente de un problema y analizando el algoritmo a diseñar para su resolución. Este análisis y diseño serán completamente independiente del tipo de herramienta a utilizar, a posteriori, para su implementación, pudiendo incluso utilizar mecanismo no en línea. Por otro lado, la Modalidad B, emplea un enfoque basado en herramientas tecnológicas que sirven para poner en práctica algunos ejemplos de pensamiento computacional. Este enfoque permitirá que el alumno aprenda, de forma más autónoma y mediante mecanismos de prueba y error, a utilizar tecnología educativa disponible para el desarrollo del pensamiento computacional.

Para la aplicación del entrenamiento se han diseñado dos grupos que siguen diferentes metodologías:

- Grupo de aprendizaje guiado (Modalidad A): en esta modalidad el alumnado lleva a cabo un curso en Code.org, en el que se estudian conceptos como condicionales y funciones. En los retos siempre hay más de una solución válida, sin embargo, es posible que una sea mejor que otra, es decir, que emplee menos código. En dichos casos, la plataforma lo indica y se anima a los alumnos a conseguir la mejor solución. Además, se utiliza Scratch, programa elaborado por el Instituto tecnológico de Massachusetts (MIT) en 2013, para simular el juego del Pong, pero de manera horizontal. El juego consiste en mantener una bola que rebota en todos los bordes, menos en el de la parte inferior, ya que, si llega hasta ahí, se pierde. Para evitarlo, se cuenta con una barra que se mueve de manera horizontal y en la cual la bola rebota. De manera adicional, se propone la incorporación de mejoras como las vidas (la bola puede rebotar una cantidad de veces en el borde inferior), el tiempo de juego o la dificultad (la bola aumenta la velocidad según el tiempo de juego).

- Grupo de aprendizaje autónomo o por descubrimiento (Modalidad B): en esta modalidad se hace uso del robot mBot. Este robot cuenta con dos ruedas y una serie de sensores, entre ellos uno de ultrasonido para detectar la distancia a la que se encuentra de distintos objetos, y otro que permite detectar si se halla sobre un color blanco o negro. En la actividad propuesta, se hace uso de los dos. Para ello, los alumnos programan el robot utilizando mBlock, un programa que combina los lenguajes de programación Scratch, basado en bloques, y Python, para que este recorra un circuito, es decir que no se salga de las líneas negras, siendo capaz de girar al lado correspondiente para corregir la dirección. Además, con el sensor de ultrasonidos, tienen que hacer que el robot se pare si encuentra algún obstáculo.

Pensamiento computacional: ¿Una nueva forma de entrenar la memoria de trabajo? Sanabria, Rodríguez, Zerpa, Prieto y Alonso.

Página 5 de 16 
Ambas modalidades de entrenamiento están diseñadas de tal forma que permitan impartirse en un total de 10 horas, distribuidas en función de la disponibilidad de los centros.

\section{Diseño}

Para este trabajo se ha establecido un diseño cuasiexperimental formado por dos grupos: un grupo guiado y grupo de aprendizaje autónomo. Posteriormente, se llevó a cabo una subdivisión dentro de cada grupo, dividiéndose en participantes con alta o baja memoria de trabajo en función de los resultados obtenidos en la prueba de memoria de trabajo verbal de Siegel y Ryan (1989).

\section{Participantes}

La muestra seleccionada para este trabajo está formada por 49 alumnos de $2^{\circ}$ de la ESO de tres centros de la isla de Tenerife, de los cuales un $33 \%$ eran mujeres y un $67 \%$ hombres. Las edades están comprendidas entre los 13 y los 15 años ( $M=13,71, \mathrm{SD}=0,70)$. Los participantes se distribuyen en dos grupos: grupo de aprendizaje guiado y grupo de aprendizaje autónomo, quedando reflejado en la Tabla 1 el número de participantes en cada condición.

Tabla 1.

\begin{tabular}{ccc}
\hline Distribución de hombres y mujeres en los grupos & \\
\hline & & Sexo \\
\hline Grupo & Hombres & Mujeres \\
\hline Guiado & 15 & 10 \\
Autónomo & 18 & 6 \\
Total & 33 & 16 \\
\hline
\end{tabular}

\section{Instrumentos}

Para la evaluación de la memoria de trabajo se han utilizado diferentes instrumentos, creándose una batería de tests. Esta batería está formada por cuatro subtests extraídos de la batería de memoria TOMAL elaborada por Reynolds y Bigler (1994), adaptada al español por Goikoetxea (2001) y el test de memoria de trabajo verbal de Siegel y Ryan (1989). A continuación se describen cada uno de los instrumentos empleados:

Test de Dígitos en orden directo: En esta prueba se le presenta al participante una secuencia de números y éste debe repetirla en el mismo orden en el que se le ha presentado. Hay dos ítems para cada amplitud, comenzando con una amplitud dos dígitos e incrementándose progresivamente el número de elementos por ítem. En total la prueba está formada por dieciocho ítems. Se termina de aplicar esta prueba cuando el participante comete tres errores seguidos.

Pensamiento computacional: ¿Una nueva forma de entrenar la memoria de trabajo? Sanabria, Rodríguez, Zerpa, Prieto y Alonso.

Página 6 de 16 
Test de dígitos en orden inverso: En esta prueba se le presenta al participante una secuencia de números, la cual tiene que repetir pero en orden inverso al que se le han presentado. Se comienza con una amplitud de dos dígitos, que se van incrementando progresivamente a medida que avanza la prueba, existiendo dos ítems para cada nivel de amplitud. Se termina de aplicar la prueba cuando el participante comete tres errores seguidos.

Test de letras en orden inverso ( $\mathbf{L i})$ : Esta prueba es una tarea que es similar a la dígitos inversos, pero este caso se lleva a cabo con elementos lingüísticos. Se le presenta una secuencia de letras en orden directo y el participante debe repetirla, pero en orden inverso al que se le han presentado. La prueba contiene dos ítems para cada nivel de amplitud, comenzando con una amplitud de dos elementos que se va incrementando progresivamente. Se termina de aplicar la prueba cuando los participantes cometen tres errores seguidos.

Test de memoria visual Abstracta (MSV): Esta prueba permite medir el recuerdo inmediato de figuras abstractas. Se le presenta una figura al participante durante cinco segundos, posteriormente se pasa a otra lámina en la que hay seis opciones, en las que el participante debe seleccionar la figura que se corresponde con la que se le presentó anteriormente. Está compuesta por treinta y seis ítems y a medida que se avanza en la prueba se va incrementando el nivel de complejidad de las figuras. Se termina de aplicar la prueba cuando el participante comete tres errores en cinco ítems.

Test de memoria de trabajo verbal: Esta prueba es una adaptación de la prueba de Daneman y Carpenter (1980) elaborada por Siegel y Ryan en (1989). La prueba está formada por cinco niveles, que se corresponden con la amplitud de memoria. Cada nivel cuenta con tres ensayos en los que se le presentan frases a las que les falta la última palabra. El participante debe decir la palabra que falta basándose en el contenido de la frase presentada y una vez ha completado todas las frases de ese ensayo debe repetirlas en el mismo orden en el que las dijo. La prueba comienza con una amplitud de dos palabras y por tanto con dos frases a completar. Se termina de aplicar esta prueba cuando el participante falla dos de los tres ensayos de un nivel, siendo el último nivel en el que acertó dos ensayos su amplitud de memoria.

Las fiabilidades test-retest de las pruebas empleadas en este trabajo oscilan entre los valores 0.70 y 0.86 .

\section{Procedimiento}

En un primer lugar se contactaba con los centros que se habían inscrito en ediciones anteriores del proyecto propuesto por el equipo de "Piens@, computacion@LLmente" de la Universidad de la Laguna, con la intención de saber si estaban interesados en participar.

Una vez obtenida la lista de colegios que querían colaborar, la participación se llevaba a cabo con dos grupos de segundo de la ESO de cada centro. A cada grupo, se les 
asignó una modalidad de entrenamiento conformándose así los grupos de aprendizaje guiado y el grupo de aprendizaje autónomo. En la primera sesión, se les administró la batería de pruebas de manera individual a cada uno de los participantes. Una vez finalizadas las sesiones de entrenamiento, se les volvió a administrar las mismas pruebas a los participantes siguiendo el mismo procedimiento que la primera vez, obteniéndose como resultado unas medidas pre y post entrenamiento.

Ya recogidos todos los datos, se dividió a los participantes en dos subgrupos, para todas las pruebas administradas, en función de la puntuación obtenida en la prueba de memoria verbal de Siegel y Ryan (1989). Para llevar a cabo la división se utilizó como punto de corte la media de las puntuaciones en esta prueba, la cual fue de 2,8. Por lo tanto los participantes con una puntuación inferior a 3 se clasificaron como de baja memoria de trabajo y a los participantes con una puntuación igual o mayor a tres como alta memoria de trabajo. A continuación se presenta en la Tabla 2 la distribución de participantes en función de la memoria de trabajo y el grupo.

Tabla 2.

Distribución de los participantes en los grupos en función del nivel de M.T

\begin{tabular}{ccc}
\hline & Grupo \\
\hline Nivel de M.T & Guiado & Autónomo \\
\hline Alto & 16 & 12 \\
Bajo & 9 & 12 \\
Total & 25 & 24 \\
\hline
\end{tabular}

\section{Análisis:}

En primer lugar se realizó prueba t con el fin de comprobar si los grupos de entrenamiento guiado y autónomo eran equivalentes en la medida inicial en las pruebas aplicadas. En segundo lugar, se realizaron pruebas t intra grupo entre las medidas pre test y post test de cada una de las pruebas empleadas. Una vez comprobado que los grupos no eran equivalentes en las medidas iniciales de las pruebas, se realizaron cuatro ANCOVAs, uno para cada una de las pruebas empleadas, tomando como variable dependiente la diferencia de las puntuaciones pre y post test en las distintas pruebas; siendo los factores intergrupo el nivel de memoria de trabajo y el grupo y empleándose como covariante la medida pre test de cada prueba. Todos los análisis se realizaron con el programa de análisis SSPS V.15. 


\section{Resultados}

Una vez recogidos los datos se llevaron a cabo los análisis obteniéndose los siguientes resultados:

Tabla 3 .

Descriptivos para la medida pre test de las pruebas en cada uno de los grupos

\begin{tabular}{cccc}
\hline Prueba & Grupo & Media & SD \\
\hline Dígitos & Guiado & 5,48 & 0,918 \\
directos & Autónomo & 4,96 & 0,690 \\
Dígitos & Guiado & 3,72 & 0,678 \\
Inversos & Autónomo & 3,42 & 0,717 \\
Letras & Guiado & 3,80 & 1,041 \\
Inversas & Autónomo & 3,13 & 0,612 \\
Figuras & Guiado & 22,28 & 8,478 \\
Abstractas & Autónomo & 16,08 & 6,467
\end{tabular}

En cuanto a los resultados obtenidos en la prueba t para comprobar que los grupos son equivalentes en las medidas iniciales de las pruebas empleadas, se obtuvo que resultó significativa la comparación entre las medidas iniciales de los grupos guiado y autónomo para la prueba de dígitos directos $\mathrm{t}(44,484)=2,254, p<0.05$; no siendo equivalentes, por lo tanto, en esta medida. Por otro lado, en la prueba de letras inversas, también resultó significativa la comparación entre las medidas iniciales de ambos grupos t $(39,121)=$ 2,708, $p<0,01$ así como la comparación de las medidas iniciales en la prueba de figuras abstractas $\mathrm{t}(44,753)=2,884 p<0,01$; indicando por tanto que los grupos no son equivalentes en las medidas iniciales de estas pruebas. Sin embargo, en la comparación para la prueba de dígitos inversos no se encontraron diferencias en las medidas iniciales entre ambos grupos $\mathrm{t}(47)=1,522, \mathrm{p}>0.05$; siendo por tanto equivalentes las medias iniciales en esta prueba.

\section{Tabla 4.}

\begin{tabular}{cccc}
\hline \multicolumn{3}{c}{ Descriptivos de las pruebas en las medida pre test y post test en las diversas pruebas } \\
\hline Prueba & Medida & Media & SD \\
\hline Dígitos & Pre test & 5,22 & 0,848 \\
directos & Post test & 5,49 & 1,023 \\
Dígitos & Pre test & 3,57 & 0,707 \\
Inversos & Post test & 3,82 & 0,950 \\
Letras & Pre test & 3,47 & 0,915 \\
Inversas & Post test & 3,57 & 0,816 \\
Figuras & Pre test & 19,24 & 8,110 \\
Abstractas & Post test & 24,16 & 7,250 \\
\hline
\end{tabular}

Pensamiento computacional: ¿Una nueva forma de entrenar la memoria de trabajo? Sanabria, Rodríguez, Zerpa, Prieto y Alonso.

Página 9 de 16 
En cuanto a los resultados obtenidos en la prueba t para comprobar si el entrenamiento había sido eficaz a la hora de producir mejoras en las medidas, se encontró que los participantes mejoraron su rendimiento en la prueba de dígitos directos (ver tabla 5). Así mismo, resultó significativa la comparación de las medidas en la prueba de dígitos inversos (ver tabla 5); indicando por tanto que los participantes obtienen puntuaciones más altas tras el entrenamiento, tal y como se puede apreciar en la tabla 4. También resultó significativa la comparación de las medidas en la prueba de figuras abstractas (ver tabla 5); obteniendo los participantes un rendimiento mayor en esta prueba tras el entrenamiento. Finalmente, en la comparación de las medidas en la prueba de letras inversas no resultó significativa, no presentando mejoras tras el entrenamiento (ver tabla $5)$.

Tabla 5.

Resultados de la prueba $t$ para las comparaciones de las medidas pre y post test en cada una de las pruebas aplicadas

\begin{tabular}{lccccc}
\hline \multicolumn{2}{c}{ Comparación } & $\mathrm{t}$ & $\mathrm{gl}$ & $\begin{array}{c}\text { Significació } \\
\mathrm{n}\end{array}$ & $\delta$ \\
\hline Dígitos directos & Pre vs. Post & $-2,100$ & 48 & $0,041^{*}$ & 0,29 \\
Dígitos inversos & Pre vs. Post & $-2,283$ & 48 & $0,027^{* *}$ & 0,30 \\
Letras inversas & Pre vs. Post & $-0,742$ & 48 & 0,462 & 0,12 \\
Figuras abstractas Pre vs. Post & $-4,396$ & 48 & $0,000^{* * * *}$ & 0,64 \\
\hline
\end{tabular}

Debido a las diferencias encontradas entre el grupo guiado y autónomo en las medidas iniciales de algunas pruebas, se llevaron a cabo varios ANCOVAs para controlar el efecto de estas diferencias iniciales, tomando como variables dependientes las diferencias de las medidas pre y post test en cada prueba y como covariante las puntuaciones pre de cada una de las pruebas; los resultados obtenidos fueron los siguientes:

En cuanto a los resultados obtenidos en el ANCOVA para la medida de dígitos directos, en los que se ha comentado que existen diferencias significativas entre las puntuaciones antes y después (ver tabla 5); se obtuvo que el efecto de la variable grupo no resultó significativo (ver tabla 6); indicando por tanto que no hay diferencias entre los grupos guiado y autónomo en el incremento de las puntuaciones para esta prueba. Así mismo, tampoco resultó significativo el efecto de la variable nivel de memoria de trabajo (ver tabla 6), presentando un incremento similar los participantes un nivel de memoria de trabajo alto y bajo. Finalmente, tampoco resultó significativa la interacción de la variable grupo x nivel de memoria de trabajo (ver tabla 6).

Pensamiento computacional: ¿Una nueva forma de entrenar la memoria de trabajo? Sanabria, Rodríguez, Zerpa, Prieto y Alonso.

Página 10 de 16 
Tabla 6.

Resultados del ANCOVA para el incremento de las puntuaciones en la prueba de dígitos directos

\begin{tabular}{lcccc}
\hline \multicolumn{1}{c}{ Efecto } & gl & F & Significación & $\eta^{2}$ \\
\hline Pre_dígitos directos & 1 & 4,100 & $0,049^{*}$ & 0,085 \\
Grupo & 1 & 0,001 & 0,982 & 0,000 \\
Nivel MT & 1 & 0,001 & 0,976 & 0,000 \\
Grupo x Nivel MT & 1 & 3,072 & 0,087 & 0,065 \\
Error & 44 & & &
\end{tabular}

En cuanto a los resultados obtenidos en el ANCOVA para la medida de dígitos inversos, en los que ya se ha comentado que no existen diferencias entre las puntuaciones antes y después (ver tabla 5). Se observa que esta ausencia de diferencias se mantiene con independencia del grupo y del nivel de memoria de trabajo (ver tabla 7).

Tabla 7.

Resultados del ANCOVA para el incremento de las puntuaciones en la prueba de dígitos inversos

\begin{tabular}{lcccc}
\hline \multicolumn{1}{c}{ Efecto } & $\mathrm{gl}$ & $\mathrm{F}$ & Significación & $\eta^{2}$ \\
\hline Pre_dígitos inversos & 1 & 1,653 & 0,205 & 0,036 \\
Grupo & 1 & 1,936 & 0,171 & 0,042 \\
Nivel MT & 1 & 0,597 & 0,444 & 0,013 \\
Grupo x Nivel MT & 1 & 1,301 & 0,260 & 0,028 \\
Error & 44 & & & \\
\hline
\end{tabular}

En el ANCOVA para el incremento de las puntuaciones en la medida de letras inversas, que como ya se ha comentado resultó significativo (ver tabla 5), se obtuvo que no resultó significativo el efecto de la variable grupo (ver tabla 8). Así mismo, tampoco resultó significativo el efecto de la variable nivel de memoria de trabajo ni la interacción de la variable grupo x nivel de memoria de trabajo (ver tabla 8).

Tabla 8.

\begin{tabular}{lcccc}
\hline $\begin{array}{l}\text { Resultados del ANCOVA para el incremento de las puntuaciones en la prueba } \\
\text { de letras inversas }\end{array}$ & gl & $\mathrm{F}$ & Significación & $\eta^{2}$ \\
\hline \multicolumn{1}{c}{ Efecto } & 1 & 28,683 & $0,000^{* * *}$ & 0,394 \\
\hline Pre_letras inversas & 1 & 0,465 & 0,499 & 0,010 \\
Grupo & 1 & 2,972 & 0,092 & 0,063 \\
Nivel MT & 1 & 1,697 & 0,199 & 0,036 \\
Grupo x Nivel MT & 44 & & & \\
Error & & & & \\
\end{tabular}

Pensamiento computacional: ¿Una nueva forma de entrenar la memoria de trabajo? Sanabria, Rodríguez, Zerpa, Prieto y Alonso. 
En relación con los resultados obtenidos en el ANCOVA para la medida de figuras abstractas, que como ya se vió anteriormente, se obtuvieron diferencias significativas entre las medidas antes-después (ver tabla 5); no resultó significativo el efecto de la variable grupo, no encontrándose diferencias entre los participantes del grupo guiado y los del grupo autónomo en el incremento de las puntuaciones en esta medida (ver tabla 10). Sin embargo, el efecto de la variable memoria de trabajo obtuvo una significación marginal (ver tabla 10); presentando un mayor incremento los participantes con un nivel de memoria de trabajo alto que los participantes con un nivel de memoria de trabajo bajo (ver tabla 9).

\section{Tabla 9}

\begin{tabular}{ccc}
\hline \multicolumn{2}{l}{ Descriptivos para el efecto nivel de memoria de trabajo } \\
\hline Nivel de memoria de trabajo & Media & SD \\
\hline Alto & 22,6 & 8,66 \\
Bajo & 20,5 & 7,05 \\
\hline
\end{tabular}

Finalmente, no resultó significativa la interacción grupo x nivel de memoria de trabajo para la medida de figuras abstractas (ver tabla 10).

Tabla 10.

Resultados del ANCOVA para el incremento en las puntuaciones en la prueba de figuras abstractas

\begin{tabular}{lcccc}
\hline \multicolumn{1}{c}{ Efecto } & $\mathrm{gl}$ & $\mathrm{F}$ & Significación & $\eta^{2}$ \\
\hline Pre_figuras Abstractas & 1 & 19,970 & $0,000^{* * *}$ & 0,312 \\
Grupo & 1 & 0,151 & 0,700 & 0,003 \\
Nivel MT & 1 & 3,850 & $0,056^{*}$ & 0,080 \\
Grupo x Nivel MT & 1 & 1,458 & 0,234 & 0,031 \\
Error & 44 & & & \\
\hline
\end{tabular}

\section{Discusión}

La memoria de trabajo es esencial a la hora de realizar cualquier tipo de tarea, ya que nos permite retener la información durante un periodo determinado de tiempo permitiendo integrarla y manipularla para resolver una determinada tarea o problema. Por esta razón, son muy interesantes los entrenamientos que permitan mejorar la memoria de trabajo, ya que se ha visto que tiene un papel importante dentro de cualquier proceso de aprendizaje (López, 2011). Además, se ha demostrado que puede ser un buen predictor, en los primeros años de escolarización, del éxito académico en etapas posteriores (Packiam y Alloway, 2010).

En este trabajo se ha empleado un entrenamiento basado en pensamiento computacional con el fin de comprobar dos hipótesis: la primera hipótesis de trabajo postula que el entrenamiento basado en pensamiento computacional es capaz de producir

Pensamiento computacional: ¿Una nueva forma de entrenar la memoria de trabajo? Sanabria, Rodríguez, Zerpa, Prieto y Alonso.

Página 12 de 16 
mejoras en las medidas de memoria de trabajo. Por otro lado, la segunda hipótesis plantea que los participantes con un nivel alto de memoria de trabajo se benefician más del entrenamiento que los participantes con un nivel bajo de memoria de trabajo.

En relación con la primera hipótesis planteada anteriormente, se ha obtenido que el entrenamiento basado en pensamiento computacional produce mejoras tanto en las medidas de dígitos directos, como en las de dígitos inversos y figuras abstractas. Sin embargo, en la prueba de letras inversas no se produjeron mejoras en el rendimiento. Estas mejoras en el rendimiento se producen independientemente del grupo en el que se encuentren los participantes, es decir, el entrenamiento que recibe el grupo guiado; el cual emplea una metodología en la que se guía a los participantes en proceso el aprendizaje es igual de eficaz a la hora de mejorar las puntuaciones en las pruebas de memoria de trabajo que el entrenamiento que recibe el grupo autónomo; el cual emplea una metodología basada en el auto descubrimiento.

Por otro lado, se llevaron a cabo cuatro Ancovas, uno para cada prueba de memoria de trabajo, empleando como covariante la medida inicial de cada prueba y como variable dependiente la diferencia de las puntuaciones antes-después de cada prueba. Este tipo de análisis se realizó debido a que se comprobó que los grupos no eran equivalentes en las medidas iniciales de las pruebas; si no se corrigen estas diferencias iniciales, podrían aparecer efectos significativos a la hora de comparar los dos grupos de entrenamiento, que no se deban a la variable tipo de entrenamiento, sino que podrían estar afectadas por las diferencias en las medidas iniciales.

Una vez se controló este problema, se obtuvo que no había diferencias en el rendimiento de los participantes de los grupos guiado y autónomo. Por lo que las dos metodologías de entrenamiento son igual de eficaces de cara a producir mejoras en las medidas de memoria de trabajo empleadas.

En relación con la segunda hipótesis, la cual postulaba que los participantes con un nivel de memoria de trabajo alto se benefician más del entrenamiento que los participantes con un nivel de memoria de trabajo bajo, se ha encontrado que el entrenamiento produce un incremento de las puntuaciones similar en los participantes con un nivel de memoria de trabajo alto y bajo en las pruebas de dígitos directos, dígitos inversos y letras inversas; no existiendo por tanto, diferencias en el incremento de las puntuaciones en función del nivel de memoria de trabajo para las pruebas mencionadas anteriormente. Sin embargo, sí se encontró que los participantes con un nivel de memoria de trabajo alto tenían un mejor rendimiento tras el entrenamiento que los participantes con un nivel de memoria de trabajo bajo en la prueba de figuras abstractas. Por otro lado, no se encontraron diferencias en el incremento de las puntuaciones de los participantes del grupo guiado y del grupo autónomo en ninguna de las pruebas de memoria de trabajo que se han empleado en este trabajo.

En resumen, se cumple la primera hipótesis de trabajo, ya que el entrenamiento, independientemente de la metodología que se emplee produce mejoras en las diferentes 
medidas de memoria de trabajo, menos para la prueba de letras inversas. Este hecho favorece la mejora de la resolución problemas ya que la memoria de trabajo es la habilidad cognitiva que permite retener una información durante un periodo de tiempo, integrar y manipularla de cara a producir una nueva(Solaz y Sanjosé, 2008). Según Zapata (2015) la resolución de problema es uno de los componentes del pensamiento computacional. Al entrenar esta capacidad, se ve una mejoría significativa en la memoria de trabajo que podría estar asociada a este tipo de entrenamiento.

Por otro lado, no se han encontrado datos suficientes que apoyen la segunda hipótesis, debido a que los participantes con un nivel de memoria de trabajo alto presentan un rendimiento similar al de los participantes con nivel de memoria de trabajo bajo en la mayoría de las pruebas; encontrándose solo diferencias en la prueba de figuras abstractas, en la cual los participantes con un nivel de memoria de trabajo alto presentan un incremento mayor en las puntuaciones.

Finalmente, no se han encontrado diferencias en el incremento de las puntuaciones entre el grupo guiado y autónomo, por lo que la metodología empleada en ambos es igual de eficaz de cara a producir cambios en las medidas de memoria de trabajo. Aunque estos resultados no son concluyentes, ya que hace falta que sean replicados en otros trabajos, así como que se controlen ciertas variables extrañas que pueden influir en su interpretación, y que limitan la generalización de los resultados.

Una de las principales limitaciones encontradas en este trabajo ha sido el tamaño muestral, impidiendo el acceso a una muestra lo suficientemente amplia como para que sea representativa de la población de estudio, debido al escaso tiempo con el que se contó a la hora de la recogida de los datos. Otra limitación encontrada ha sido la ausencia de un grupo control que permitiese comprobar la veracidad de que los cambios se han producido sean debido al entrenamiento y no por el paso del tiempo o por la maduración de los participantes. De cara a futuros trabajos, se podría solventar este problema ampliando el tamaño de la muestra y añadiendo un tercer grupo que no reciba ningún entrenamiento, con el fin de respaldar así los resultados obtenidos y controlar las posibles variables extrañas que podrían influir en los resultados.

Así mismo, dado que el entrenamiento produce mejoras en las diferentes medidas de memoria de trabajo en la muestra empleada, es interesante seguir trabajando en esta línea, intentando apresar y cuantificar el potencial que pueden tener los entrenamientos basados en el pensamiento computacional de cara a producir mejoras en diversos procesos cognitivos.

\section{Agradecimientos}

A todo el equipo del proyecto “Piens@ computacion@LLmente" de la Universidad de La Laguna por permitirnos participar en este proyecto y recoger los datos que han dado como resultado este trabajo.

Pensamiento computacional: ¿Una nueva forma de entrenar la memoria de trabajo? Sanabria, Rodríguez, Zerpa, Prieto y Alonso.

Página 14 de 16 
Presentación del manuscrito: 10 de enero de 2020

Fecha de aprobación: 26 de marzo de 2020

Fecha de publicación: 30 de abril de 2020

Sanabria, E., Rodríguez, N., Zerpa, A.E., Prieto y Alonso, M.A. (2020). Pensamiento computacional: ¿Una nueva forma de entrenar la memoria de trabajo? RED. Revista Educación a Distancia, 20(63). http://dx.doi.org/10.6018/red.401931

\section{Financiación}

Este trabajo no ha recibido ninguna subvención específica de los organismos de financiación en los sectores públicos, comerciales o sin fines de lucro.

\section{Referencias}

Adell, J. S., Llopis, M. A. N., Esteve, M. F. M., y Valdeolivas, N. M. G. (2019). El debate sobre el pensamiento computacional en educación. RIED. Revista Iberoamericana de Educación a Distancia, 22(1), pp. 171-186.

Aguirre, N., Cruz-Gómez, Á.J., Miró-Padilla, A., Bueichekú, E., Solozano, N., BrosetaTorres, R.,...Forn, C. (2018). El entrenamiento en memoria de trabajo mejora la eficiencia cognitiva en pacientes de esclerosis múltiple, Revista Discapacidad Clinica Neurociencia, 5(2), pp. 16-25

Baddeley, A (2000) The episodic buffer: a new component of working memory?.Trends in cognitive science. 4 (11) pp. 417-423.

Baddeley, A. y Hitch, G. (1974). Working memory. En G.A. Bower (ed.), The psychology of learning and motivation:Advances in research and theory.. Vol. 8 pp. 47-89. Nueva york: Academic Press.

Beloe, P. y Darakshan, N. (en prensa). Adaptative working memory training can reduce anxiety and depression vulnerability in adolescents. Developmental science. doi: 10.1111/desc.12831

Daneman, M. y Carpenter, P. (1980). Individual Differences in Working Memory and Reading. Journal of verbal learning and verbal behavior, 19(4), pp. 450-466.

García, F. y Mendes, A (2018) Exploring the computational thinking effects in preuniversity education, Computers in Human Behavior, 1(80), pp. 407-411.

Gonzales-Nieves, S., Fernández-Morales, F.H. y Duarte, J.E.(2018). Efecto del entrenamiento de memoria de trabajo y mindfulness en la capacidad de la memoria de trabajo y el desempeño matemático en niños de segundo grado, Revista Mexicana de investigación Educativa, 78(23), pp. 841-859

Kallia, M., \& Psycharis, S. (2017). The effects of computer programming on high school students' reasoning skills and mathematical self-efficacy and problem solving. Instructional science, 45(5), pp. 583-602.

Pensamiento computacional: ¿Una nueva forma de entrenar la memoria de trabajo? Sanabria, 
Klingberg, T., Fernerll, E., Olesen, P.J., Johnson, M., Gustafsson, P., Dahlström, K.... Westerberg, H.(2005). Computerized Training of Working Memory in Children with ADHD - A Randomized, Controlled Trial, Journal of the American Academy of Child and Adolescent Psychiatry, 44(2), pp. 177-186.

Korkmaz,Ö.(2016).The Effect of Scratch- and Lego Mindstorms Ev3-Based Programming Activities on Academic Achievement, Problem-Solving Skills and Logical-Mathematical Thinking Skills of Students, Malaysian Online Journal of Educational Sciences, 3(4), pp. 73-86.

Llorens, F. (2015) Dicen por ahí. . . que la nueva alfabetización pasa por la programación, ReVisión, 2 (8), pp. 11-14

López, M (2011) Memoria de trabajo y aprendizaje: aportes de la neuropsicología, Cuadernos de Neuropsicologia - Panamerican Journal of Neuropsychology,1(5), pp. 25-47.

Packiam, T. y Alloway, R. G. (2010) investigating the predictive roles of working memory and IQ in academic attainment, Journal of Experimental Child Psichology, 1(106), pp. 20-29.

Reynolds, C. y Bigler, E. (1994). TOMAL-Test of memory and learning. Austin: Pro.ed.

Reynolds, C. y Bigler, E. (2001). TOMAL-Test de memoria y aprendizaje. Madrid: TEA

Román., Pérez, JC., Jiménez C. (2017) Which cognitive abilities undelie computational thinking? Criterion validity of the computational thinking test. Computer in human behavior, 1(72), pp. 678-691.

Scherer, R., Siddiq, F., \& Sánchez Viveros, B. (2018). The cognitive benefits of learning computer programming: A meta-analysis of transfer effects. Journal of Educational Psychology. Advance online publication, pp. 1-29.

Siegel, L.S. y Ryan, E.B. (1989). The development of working memory in normally achieving and subtypes of learning disabled children. Child Development, 60(4), pp. 973-980.

Solaz,J \& Sanjose, V. (2008). Conocimientos y procesos cognitivos en la resolución de problemas de ciencias: consecuencias para la enseñanza, Magis. Revista internacional de investigación en educación, 1(1), pp.147-162

Wing, J. (2006). Computational thinking. It represents a universally aplicable attitude and skill set everyone, not just computer scientist, would be eager to learn and use. Comunications of the ACM., 49 (3) pp. 33-35.

Zapata, M. (2015): Pensamiento computacional: Una nueva alfabetización digital. Revista de educación a distancia. 46 (4). pp.1-47. doi: 10.6018/red/46/4

Pensamiento computacional: ¿Una nueva forma de entrenar la memoria de trabajo? Sanabria, Rodríguez, Zerpa, Prieto y Alonso. 